\title{
Adult honeybees (Apis mellifera L.) abandon hemocytic, but not phenoloxidase-based immunity
}

\author{
Martin R. Schmid ${ }^{\mathrm{a}, 1,2}$, Axel Brockmann ${ }^{\mathrm{a}, 1}$, Christian W.W. Pirk ${ }^{\mathrm{a}, \mathrm{b}}$, David W. \\ Stanley ${ }^{c}$ and Jürgen Tautz ${ }^{\mathrm{a}}$ \\ a BEEgroup, Biozentrum Universität Würzburg, Am Hubland, D-97074 Wuerzburg, \\ Germany \\ b Department of Zoology and Entomology, University of Pretoria, Pretoria 0002, South \\ Africa \\ c Biological Control of Insects Research Laboratory, USDA/Agricultural Research \\ Service, 1503 S. Providence, Columbia, MO 65203, USA
}

\section{Abstract}

Hemocytes and the (prophenol-) phenoloxidase system constitute the immediate innate immune system in insects. These components of insect immunity are present at any postembryonic life stage without previous infection. Differences between individuals and species in these immune parameters can reflect differences in infection risk, life expectancy, and biological function. In honeybees which show an age-related division of labor within the worker caste, previous studies demonstrated that foragers show a strongly reduced number of hemoctyes compared to the younger nurse bees. This loss of immune competence has been regarded advantageous with respect to an already high mortality rate due to foraging and to redistribution of energy costs at the colony level. Based on the idea that abandoning hemocytes in all adults would be a reasonably direct regulatory mechanism, we posed the hypothesis that abandoning hemocytic immunity is not restricted to worker honeybees. We tested our hypotheses by performing a comprehensive analysis of hemocyte number and phenoloxidase (PO)-activity levels in immunologically naive workers, queens, and drones. We found that in all three adult phenotypes hemocyte number is dramatically reduced in early adult life. In contrast, we found that the dynamics of PO-activity levels have sex and caste-specific characteristics. In workers, PO activity reached a plateau within the first week of adult life, and in queens enzyme levels continuously increased with age and reached levels twice as high as those found in workers. PO-activity levels slightly declined with age in drones. These data support our hypothesis, from which we infer that the previously reported reduction of hemocyte in foragers is not worker specific but represents a general phenomenon occurring in all honeybee adult phenotypes.

\section{Article Outline}

1. Introduction

2. Material and methods 
2.1. Bees

2.2. Hemocyte number and phenoloxidase (PO) activity

2.3. Data analysis

3. Results

3.1. Changes in hemocyte number in workers, queens, and drones

3.2. Changes in PO activity in workers, queens, and drones

4. Discussion

Acknowledgements

References

\section{Introduction}

Insect innate immunity is usually resolved into two broad categories: cellular and humoral immunity (Gillespie et al., 1997). Cellular immunity involves direct interactions between circulating hemocytes and invading organisms (Lavine and Strand, 2002). These defense interactions include phagocytosis, nodulation and, for invaders too large for cellular internalization, encapsulation. Nodulation as well as encapsulation is often accompanied by melanization, catalyzed by the (prophenol-) phenoloxidase (PO) (Söderhall and Cerenius, 1998). Cellular immune reactions and prophenoloxidase (proPO) activation are launched immediately after an infection is detected and are responsible for clearing most infecting microbial challengers from insect circulation within the first hours (Dunn and Drake, 1983). Both reactions make up the insects' constitutive immune system, which is present at any post-embryonic life stage without previous infection (Brown et al., 2003).

Although maintaining immune competence is vitally important for the organism, it also is a very costly physiological function (Schmid-Hempel, 2003). In insects, physiological reallocation of costs between immune responses and survival (Moret and SchmidHempel, 2001), reproductive behavior (Rolff and Siva-Jothy, 2002), foraging (König and Schmid-Hempel, 1995), and learning (Mallon et al., 2003) have been documented. While it is generally agreed that immune functions can be traded off against other functions (Zuk and Stoehr, 2002), the underlying mechanisms and evolutionary forces driving the trade-offs remain contemporary topics (Rolff, 2001a). Regarding social insects, there may be sex and caste differences in immune capacity. Male bumblebees, Bombus terrestris, appear to be less immunocompetent than females (Gerloff et al., 2003). These findings raise the question of whether in honeybees the sexes and female castes might show differences in their constitutive immune system anticipating differences in life history and task.

Previous studies in honeybees demonstrated that foragers undergo a dramatic reduction in circulating hemocyte numbers, a reduction in hemolymph volume, and an almost total loss of the capability to form nodules in response to bacterial challenge (Wille and Rutz, 1975; Fluri et al., 1977; Bedick et al., 2001; Amdam et al., 2004). From an evolutionary perspective, hemocyte reduction in honeybee foragers may represent a colony-level strategy of trading off costs of immunity against energy reserves of the whole colony. As 
foragers already face a high mortality rate, e.g. due to predation (Neukirch, 1982), reduction of immune competence might not substantially increase mortality risk for the individual but may be beneficial to the colony, as it saves energy costs (e.g. stored food resources) needed to maintain cellular immunity. Correspondingly, loss of hemocytes may be an integral part of division of labor regulated by vitellogenin and juvenile hormone ([Amdam et al., 2004] and [Amdam et al., 2005]). Both these hypotheses imply two important predictions about dynamics of hemoctye numbers in honeybee female castes: in workers, hemocyte number should be task- and not age-dependent, and in queens, which are long-lived and show a reverse pattern of vitellogenin and juvenile hormone compared to workers (Bloch et al., 2002; Corona et al., 2007; Fluri et al., 1982), high hemocyte numbers should be maintained throughout their entire lives.

Assuming that hemocyte production and maintenance is a physiologically expensive option cost (Zuk and Stoehr, 2002) and social colonies with immense numbers of sterile workers and males would gain from a reduction of these costs, a plausible strategy would be to abandon hemocytic immunity in all individuals, if at the same time immune competence for the queen is maintained or improved. Thus, we propose the alternative hypothesis that abandoning hemocytic immunity is not restricted to worker honeybees but occurs in all adult honeybees. In addition, constitutive PO activity (Lourenco et al., 2005), which in plasma of larval lepidopterans can inactivate baculoviruses (Popham et al., 2004), might be the candidate immune function to compensate hemocyte loss, if necessary (Rolff, 2001b). In this paper, we report on the outcomes of experiments designed to test these hypotheses.

\section{Material and methods}

\subsection{Bees}

Honeybee workers, queens, and drones originated from Apis mellifera carnica L. colonies maintained at the University of Würzburg, Germany.

For the subsequent two age series, 1-day-old bees from different source colonies were paint marked and introduced into the same healthy foster colony. Samples for the two worker age series were collected over 3 weeks. For hemocyte number, we obtained measurements for $1-4,7,8,12,13,18$, and 23 days of age, and for PO-activity determinations, we obtained measurements for 1-17, 19, 22, and 24 days of age. For each day of sampling, we determined hemocyte number and PO activity for 10 individuals, and at any time point new individuals were examined. For the analysis of queens, we collected 6-month (hemocytes (H): $n=3$, PO: $n=4)$, 1-year (H: $n=4$, PO: $n=4)$, and 2-yearold (H: $n=1$, PO: $n=2)$ queens from colonies that were scheduled for artificial queen exchange. We artificially reared 1-day-old queens (H: $n=8$, PO: $n=7)$.

Precocious foragers and over-aged nurses were produced using the single cohort colony (SCC) procedure (Robinson et al., 1989). We collected precocious foragers and age-right nurses from 5 to 11 days of age, and age-right foragers and over-aged nurses from 23 to 28 days of age. Workers were identified as foragers, if they showed visual signs of pollen and nectar foraging (Jassim et al., 2000). Nurses were identified by briefly removing 
brood combs from the colony and observing individual bees that dipped their heads successively into several cells containing larvae. We confirmed our identifications as ageright and over-aged nurses have significantly more developed hypopharyngeal glands than precocious and age-right foragers of the respective age (Kruskal-Wallis: $H_{1,92}=64.22, P<0.0001$; Mann-Whitney $U$ test: $P<0.001$, each $U \leqslant 6$ ).

\subsection{Hemocyte number and phenoloxidase (PO) activity}

Hemocyte number was estimated according to Gupta (1986) using a hemocytometer (Superior, Marienfeld, Germany) and phase-contrast microscopy (Axiophot Zeiss, Jena). Cell counts were done 5-10 min after filling the hemocytometer.

For the measurement of PO-activity levels, thoraces of individual bees were homogenized in liquid nitrogen and then incubated in $1 \mathrm{ml}$ of ice-cold bee saline for $1 \mathrm{~h}$ at room temperature and an additional $24 \mathrm{~h}$ at $4{ }^{\circ} \mathrm{C}$. After centrifugation, $20 \mu \mathrm{l}$ of the supernatant were mixed with $140 \mu \mathrm{l}$ of distilled water, $20 \mu \mathrm{l}$ of sodium phosphate buffer, and $20 \mu \mathrm{l}$ l-Dopa (Sigma D-9626, $4 \mathrm{mg} / \mathrm{ml}$ deionized water) as a substrate. PO activity at $30{ }^{\circ} \mathrm{C}$ was measured at $490 \mathrm{~nm}$ for $20 \mathrm{~min}$ in 1-min intervals (MRX Microplate Absorbance Reader, Dynex Technologies). For each bee, we performed two independent measurements and determined an average O.D. $\left(V_{\max }\right)$ for the two reactions.

\subsection{Data analysis}

A general linear model (GLM) design was used to obtain weighted regression equations measuring number of hemocytes and PO levels at different ages for three castes of adult honeybees. A completely randomized design (CRD) with unequal replication was used to evaluate mean differences in hemocytes and PO levels between four single cohort groups of adult honeybees.

Weighted regression equations for Fig. 1 of the mean number of hemocyte cells as a function of age were obtained for workers age series 1 , workers age series 2 , queens, and drones. Weighted regression equations for Fig. 2 of the mean amount of $\mathrm{PO}$ as a function of age were obtained for workers age series 1 , workers age series 2 , queens, and drones. The mean number of cells and mean PO amounts at certain ages were weighted by $1 /$ variance. GLM F-tests were performed to determine if there were differences between regression equations for age series 1 and age series 2 workers in number of hemocytes and in amount of $\mathrm{PO}$ as a function of age at $P=0.05$. 

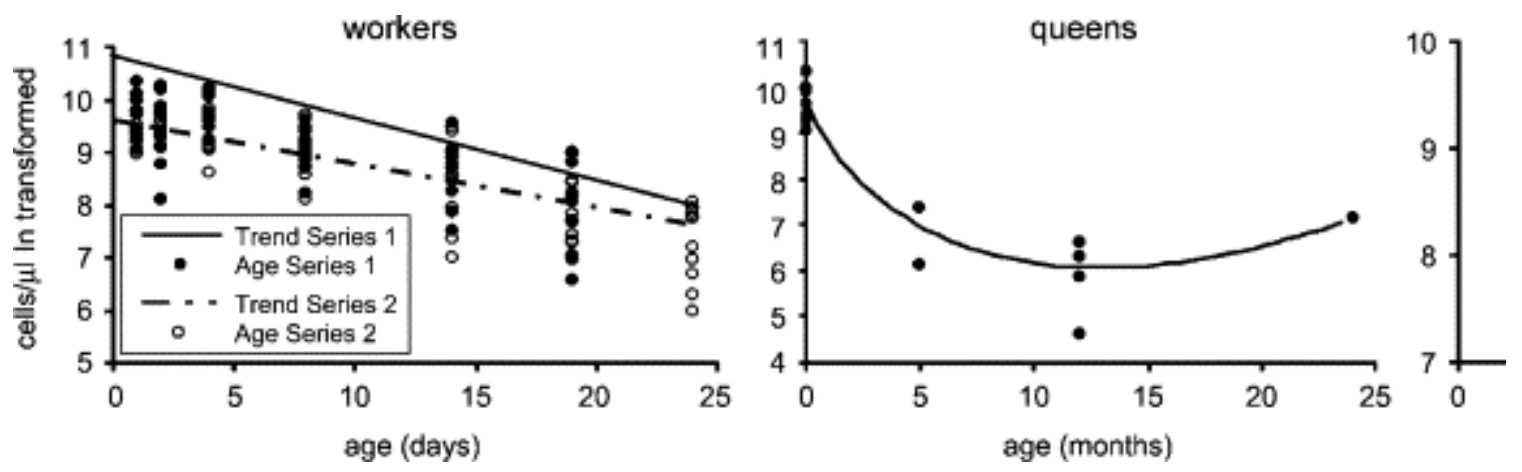

Fig. 1. Changes of hemocyte number in worker, queen, and drone honeybees in relation to age.
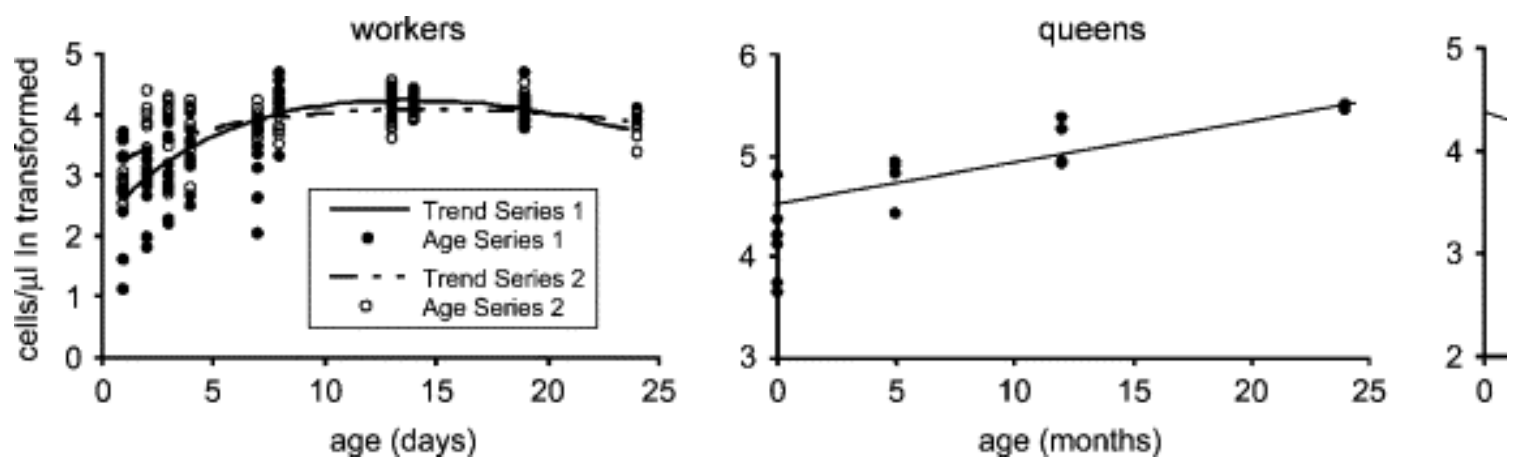

Fig. 2. Changes of phenoloxidase activity in worker, queen, and drone honeybees in relation to age.

Single-factor analysis of variance (ANOVAs) for unequal replication were used to analyze differences in mean number of hemocytes and mean PO amounts between four single cohort groups of adult honeybees (age-right nurses, over-aged nurses, age-right foragers, and precocious foragers). Levene's homogeneity of variance test was performed on hemocyte and PO data to check if data transformation was needed before ANOVAs could be performed. All analyses were performed on transformed data where necessary, but raw data is presented for ease of interpretation. If a significant $F$-test statistic (at $P$ K 0.05) was obtained from an ANOVA, differences of least squares means was used as the multiple comparison procedure for determining cohort group differences. Statistical analyses were performed using SAS/STAT ${ }^{\circledR}$ (version 9.1.3, 2002-2003, SAS Institute Inc., Cary, NC, USA) and TableCurve 2D (version 5.00, Automated Curve Fitting and Equation Discovery, 2000, AISN Software Inc., Mapleton, OR, USA). 


\section{Results}

\subsection{Changes in hemocyte number in workers, queens, and drones}

In both worker age series, hemocyte number continuously decreased with age (Fig. 1; linear regression analysis: age series 1 adjusted $R^{2}=0.89, P<0.002$; age series 2 adjusted $\left.R^{2}=0.94, P=0.0001\right)$. Similar to workers, hemocyte number in queens and drones also declined sharply, and non-linearly, with age, (queens: $R^{2}=0.9997, P<0.0001$; drones: $R^{2}=0.89, P<0.025$; Fig. 1).

In the SCC experiment, hemocyte numbers in older nurses and foragers were much smaller compared to their younger counterparts $(P \leqslant 0.05)$, indicating that the overall hemocyte decline is age- but not task-dependent. Although numbers of hemocytes were already small in over-aged nurses and age-right foragers, over-aged nurses have a significantly higher number of hemocytes compared to foragers of similar age (Fig. 3a).
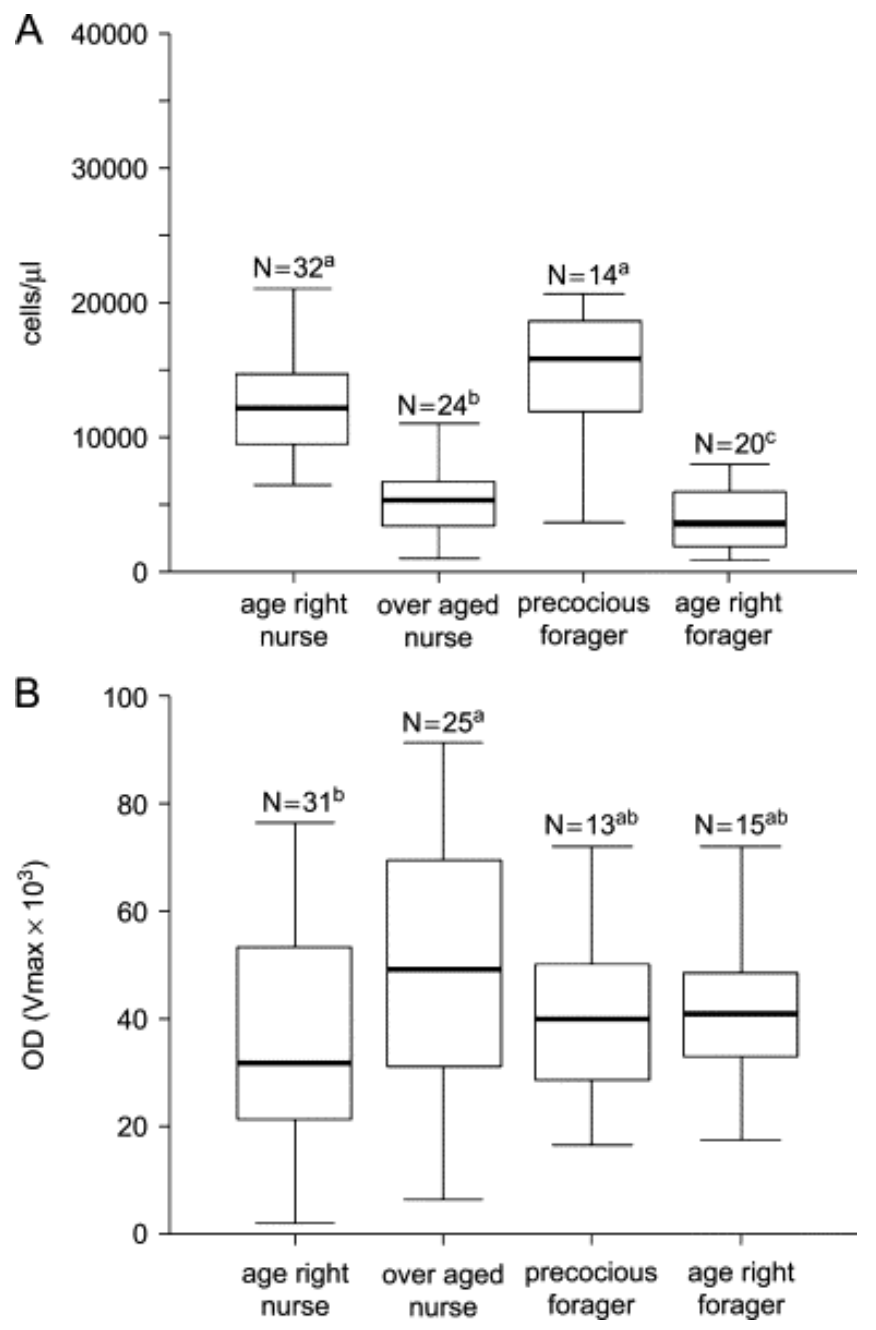

Fig. 3. Hemocyte number and phenoloxidase activity in workers of a single cohort colony: age-right nurses (5-11 days), over-aged nurses (23-28 days), precocious foragers 
(5-11 days), and age-right foragers (23-28 days). Values with the same superscript letter are not significantly different.

\subsection{Changes in PO activity in workers, queens, and drones}

PO activity in workers increased with age and reached a plateau within the first week of adult life (Fig. 2; age series 1: $R^{2}=0.81, P<0.002$; age series $2: R^{2}=0.86, P<0.001$ ). In queens, PO activity increased with age (Fig. 2), however, unlike our findings with workers, PO activity continued to increase with age $\left(R^{2}=0.94, P<0.01\right)$. PO-activity levels in 1-2-year-old queens were twice as high as those found in old workers. In contrast to the female castes, PO-activity levels in drones declined with age $\left(R^{2}=0.999, P<0.001\right)$. In the SCC experiment, PO activity was significantly increased in over-aged nurses compared to age-right nurses $(P \leqslant 0.05$; Fig. $3 b)$. All other combinations did not yield significant differences.

\section{Discussion}

The outcomes of our experiments support our hypotheses that abandoning hemocytic immunity is not restricted to worker honeybees but that all adults undergo hemocyte loss but do not loose PO activity. Several points are germane. First, hemocyte populations declined with increasing age in workers, queens, and drones. Second, constitutive PO activity increased with age in workers and queens and declined slightly throughout the 30-day experiments in drones. Third, the SCC experiment revealed that the changes in hemocyte populations were related to age and not to task. We infer our data are consistent with the idea that adult honeybees of all castes abandon hemocytic immunity while retaining constitutive PO-based immunity.

Previous studies on honeybee immunity demonstrated that foragers show a pronounced reduction in hemocyte number (Wille and Rutz, 1975; Fluri et al., 1977; Bedick et al., 2001; Amdam et al., 2004) and an almost total loss of the capability to form nodules in response to bacterial challenge (Bedick et al., 2001). Although survival experiments have not been reported, these results have been taken as evidence that foragers endure a severe reduction in immune competence. Our measurements in workers, queens, and drones revealed that all three adult castes undergo a strong reduction in hemocyte number during adult life. This indicates that hemocyte loss is not worker specific.

Our findings with over-aged nurses and precocious foragers indicate that the loss of hemocytes in workers is age- rather than task-dependent, and thus is not regulated by juvenile hormone and vitellogenin orchestrating the physiological changes during nurse forager transition. Nevertheless, hemocyte loss in adult honeybees might be regulated by endocrine factors. Age-related hemocyte loss has been recorded in other insect species (Hillyer et al., 2005; Stanley and Park, unpublished data). For one example, Stanley and Park (unpublished data) recorded a steady decline in hemocyte populations in adult males of the cricket Gryllus firmus beginning after they reached the age of 3 weeks. This may be due to a decline in the ability to replace dying hemocytes with age. The kinetics of hemocyte loss in honeybees is much faster than the kinetics registered for adult male 
crickets and mosquitoes, from which we would suggest the honeybee hemocyte loss is a regulated event. The regulating mechanism remains another research frontier.

The evolutionary forces at work in adult honeybee hemocyte loss appear to be quite complex. If the loss of hemocytes were a sole consequence of the biological expenses of foraging flight energetics, we would not predict a hemocyte loss in queens. Queens are indispensable to honeybee colonies and we take the losses recorded for long-lived queens to indicate that hemocyte loss is not a direct trade-off for flight energetics at the level of worker bees. An alternative view is that hemocyte loss may be a trade-off at the level of the colony energy economy. This idea could be supported if the economy were taken to include energy requirements for work inside the hive and costs of reproduction as well as flight energetics.

Trading off hemocytic immune functions would be even more attractive if the trade-off does not entirely cripple honeybee immunity. Experiments with Drosophila mutants showed that the loss of any one of the three immune measures (hemocyte number, PO activity or anti-bacterial peptides) does not increase mortality rate (Braun et al., 1998). Thus, if cellular immunity is disposable to some extent, the apparent risks associated with hemocyte loss may be substantially reduced.

PO-catalyzed melanization of pathogens is a very important component of invertebrate defenses (Cerenius and Söderhall, 2004) and may well compensate for hemocyte losses. PO-activity levels increased with age in workers and queens. Particularly, we recorded very high PO-activity levels in old queens, substantially higher than levels registered for workers. In the SCC experiments, we found significantly higher PO activity in over-aged nurses compared to their age-right counterparts. We did not record a clear effect of age on PO activity in foragers, possibly because the young bees collected in this experiment were between 5 and 11 days in adult age. We suggest that at this age, workers already exhibit maximum PO-activity levels; expression of AmproPO, the transcript coding for proPO, starts in late pupal phase and continues to increase till day 4 after emergence, when it reaches a plateau (Lourenco et al., 2005). Turning to queens, PO activity did not reach a plateau phase within the first week of adult life but continuously increased with age. In drones, PO activity remained constant through the experimental period. Thus, in contrast to hemocyte numbers, PO-activity changes in adult honeybees vary between sexes and castes and correlate with differences in longevity and significance for colony survival.

We measured PO-activity levels for thorax preparations, composed of hemolymph as well as epidermal and cuticular bound PO. Preliminary studies in workers had indicated that PO-activity levels in the hemolymph increase with age; however, increases in POactivity levels were more pronounced for preparations including epidermal tissue and cuticle. Thus, it might be possible that in the absence of hemocytes and reduction of hemolymph volume, age-dependent increase in PO levels in the female castes reflects enhanced epidermal-related immune defenses. 
We cannot exclude the possibility that the decline in total hemocyte numbers might comprise a change in relative numbers of specific types of hemocytes. Kurtz (2002) considered the changes of hemocyte numbers and phagocytic capacity in the scorpionfly Panorpa vulgaris. During larval development, total hemocyte number decreased, while number of phagocytosing hemocytes increased. Adults, on the other hand, appeared to undergo decreases in phagocytic capacity, while hemocyte numbers remained much the same. It follows that changes in subpopulations of hemocytes may be quite important in understanding overall cellular immunocompetence. The slight but significant difference in hemocyte number between over-aged nurses and age-right foragers might be a hint that despite the overall hemocyte loss, there are more subtle differences in hemocyte dynamics in adult honeybees (see also Fluri et al., 1977; Amdam et al., 2005).

In a similar study to ours, (Rolff, 2001a) and (Rolff, 2001b) found that sexually mature male and female dragonflies, Lestes viridis, captured in the wild, had higher hemocyte counts and lower PO activity than their newly emerged counterparts, demonstrating for the first time that these two immune measures do not necessarily correlate with each other and might even be regulated according to specific conditions. In the case of honeybees, our study suggests that the marked drop in hemocytes is coupled with increased PO activity, indicating a programmed change in immune functions, from cellular-based to PO-based immunity.

Whole genome analysis revealed that honeybees possess only about one-third of genes involved in immune function compared to Drosophila and Anopheles (Evans et al., 2006). The authors suggested two plausible reasons for the reduction in the immune system in honeybees: (1) an enlarged social immune defense, including hygienic behavior and highly antibacterial nest environment, and (2) a limitation in the number coevolved pathogens. With respect to these hypotheses, we note that the closely related eusocial bumble bees, which differ from honeybees in their hygienic behavior and the antibacterial conditions in nests, do not show a reduction in hemocyte number with age (Doums et al., 2002; König and Schmid-Hempel, 1995). Returning to our comment that the evolutionary forces driving hemocyte loss are complex, we suggest the evolution of more complex hygienic behaviors and nest conditions (Stow et al., 2007) enabled honeybees, but not bumble bees, to trade off individual hemocytic-based defense while retaining an effective, albeit less expensive, PO-based defense system.

\section{References}

Amdam et al., 2004 G.V. Amdam, Z.L. Simoes, A. Hagen, K. Norberg, K. Schroder, O. Mikkelsen, T.B. Kirkwood and S.W. Omholt, Hormonal control of the yolk precursor vitellogenin regulates immune function and longevity in honeybees, Experimental Gerontology 39 (2004), pp. 767-773. 
Amdam et al., 2005 G.V. Amdam, A.L. Aase, S.C. Seehuus, K.M. Fondrk, K. Norberg and K. Hartfelder, Social reversal of immunosenescence in honey bee workers, Experimental Gerontology 40 (2005), pp. 939-947.

Bedick et al., 2001 J.C. Bedick, H. Tunaz, A.R. Nor Aliza, S.M. Putnam, M.D. Ellis and D.W. Stanley, Eicosanoids act in nodulation reactions to bacterial infections in newly emerged adult honey bees, Apis mellifera, but not in older foragers, Comparative Biochemistry and Physiology C 130 (2001), pp. 107-117.

Bloch et al., 2002 G. Bloch, D.L. Wheeler and G.E. Robinson, Endocrine influences on the organization of insect societies. In: D.W. Pfaff, A.P. Arnold, A.M. Etgen, S.E.

Fahrbach and R.T. Rubin, Editors, Hormones and Behavior, Academic Press, New York (2002), pp. 195-236.

Braun et al., 1998 A. Braun, J.A. Hoffmann and M. Meister, Analysis of the Drosophila host defense in domino mutant larvae, which are devoid of hemocytes, Proceedings of the National Academy of Sciences USA 95 (1998), pp. 14337-14342.

Brown et al., 2003 M.J.F. Brown, Y. Moret and P. Schmid-Hempel, Activation of host constitutive immune defence by an intestinal trypanosome parasite of bumble bees, Parasitology 126 (2003), pp. 253-260.

Cerenius and Söderhall, 2004 L. Cerenius and K. Söderhall, The prophenoloxidaseactivating system in invertebrates, Immunological Reviews 198 (2004), pp. 116-126.

Corona et al., 2007 M. Corona, R. Velarde, S. Remolina, A. Moran-Lauter, Y. Wang, K.A. Hughes and G.E. Robinson, Vitellogenin, juvenile hormone, insulin signaling, and queen honey bee longevity, Proceedings of the National Academy of Sciences USA 104 (2007), pp. 7128-7133.

Doums et al., 2002 C. Doums, Y. Moret, E. Benelli and P. Schmid-Hempel, Senescence of immune defence in Bombus workers, Ecological Entomology 27 (2002), pp. 138-144.

Dunn and Drake, 1983 P.E. Dunn and D.R. Drake, Fate of bacteria injected into naive and immunized larvae of the tobacco hornworm, Manduca sexta, Journal of Invertebrate Pathology 41 (1983), pp. 77-85.

Evans et al., 2006 J.D. Evans, K. Aronstein, Y.P. Chen, C. Hetru, J.L. Imler, H. Jiang, M. Kanost, G.J. Thompson, Z. Zou and D. Hultmark, Immune pathways and defense mechanisms in honey bees Apis mellifera, Insect Molecular Biology 15 (2006), pp. 645656.

Fluri et al., 1977 P. Fluri, H. Wille, L. Gerig and M. Lüescher, Juvenile hormone, vitellogenin and haemocyte composition in winter worker honey bees, Experientia 33 (1977), pp. 1240-1241. 
Fluri et al., 1982 P. Fluri, M. Lüscher, H. Wille and L. Gerig, Changes in weight of the hypopharyngeal gland and haemolymph titres of juvenile hormone, protein and vitellogenin in worker honey bees, Journal of Insect Physiology 28 (1982), pp. 61-68.

Gerloff et al., 2003 C.U. Gerloff, B.K. Ottmer and P. Schmid-Hempel, Effects of inbreeding on immune response and body size in a social insect, Bombus terrestris, Functional Ecology 17 (2003), pp. 582-589.

Gillespie et al., 1997 J.P. Gillespie, M.R. Kanost and T. Trenczek, Biological mediators of insect immunity, Annual Review of Entomology 42 (1997), pp. 611-643.

Gupta, 1986 A.P. Gupta, Arthropod immunocytes identification, structure, functions, and analogies to the functions of vertebrates B- and T-lymphocytes. In: A.P. Gupta, Editor, Hemocytic and Humoral Immunity in Arthropods, Wiley, New York (1986), pp. 3-59.

Hillyer et al., 2005 J.F. Hillyer, S.L. Schmidt, J.F. Fuchs, J.P. Boyle and B.M.

Christensen, Age-associated mortality in immune challenged mosquitoes (Aedes aegypti) correlates with a decrease in haemocyte numbers, Cellular Microbiology 7 (2005), pp. $39-51$.

Jassim et al., 2000 O. Jassim, Z.Y. Huang and G.E. Robinson, Juvenile hormone profiles of worker honey bees, Apis mellifera, during normal and accelerated behavioural development, Journal of Insect Physiology 46 (2000), pp. 243-249.

König and Schmid-Hempel, 1995 C. König and P. Schmid-Hempel, Foraging activity and immunocompetence in workers of the bumble bee, Bombus terrestris L., Proceedings of the Royal Society of London B 260 (1995), pp. 225-227.

Kurtz, 2002 J. Kurtz, Phagocytosis by invertebrate hemocytes: causes of individual variation in Panorpa vulgaris scorpionflies, Microscopic Research Techniques 15 (2002), pp. $456-468$.

Lavine and Strand, 2002 M.D. Lavine and M.R. Strand, Insect hemocytes and their role in immunity, Insect Biochemistry and Molecular Biology 32 (2002), pp. 1295-1309.

Lourenco et al., 2005 A.P. Lourenco, M.S. Zufelato, M.M. Bitondi and Z.L. Simoes, Molecular characterization of a cDNA encoding prophenoloxidase and its expression in Apis mellifera, Insect Biochemistry and Molecular Biology 35 (2005), pp. 541-552.

Moret and Schmid-Hempel, 2001 Y. Moret and P. Schmid-Hempel, Immune defence in bumble-bee offspring, Nature 414 (2001), p. 506.

Neukirch, 1982 A. Neukirch, Dependence of the life span of the honeybee (Apis mellifera L.) upon flight performance and energy consumption, Journal of Comparative Physiology 146 (1982), pp. 35-40. 
Popham et al., 2004 H.J.R. Popham, K.S. Shelby, S.L. Brandt and T.A. Coudron, Potent virucidal activity in larval Heliothis virescens plasma against Helicoverpa zea single capsid nucleopolyhedrovirus, Journal of General Virology 85 (2004), pp. 2255-2261.

Robinson et al., 1989 G.E. Robinson, R.E. Page, C. Strambi and A. Strambi, Hormonal and genetic control of behavioral integration in honey bee colonies, Science 246 (1989), pp. 109-112.

Rolff, 2001a J. Rolff, Bateman's principle and immunity, Proceedings of the Royal Society London B 269 (2001), pp. 867-872.

Rolff, 2001b J. Rolff, Effects of age and gender on immune function of dragonflies (Odonata, Lestidae) from a wild population, Canadian Journal of Zoology 79 (2001), pp. 2176-2180.

Rolff and Siva-Jothy, 2002 J. Rolff and M.T. Siva-Jothy, Copulation corrupts immunity: a mechanism for a cost of mating in insects, Proceedings of the National Academy of Sciences USA 99 (2002), pp. 9916-9918.

Schmid-Hempel, 2003 P. Schmid-Hempel, Variation in immune defence as a question of evolutionary ecology, Proceedings of the Royal Society of London B 270 (2003), pp. $357-366$.

Söderhall and Cerenius, 1998 K. Söderhall and L. Cerenius, Role of the prophenoloxidase-activating system in invertebrate immunity, Current Opinion in Immunology 10 (1998), pp. 23-28.

Stow et al., 2007 A. Stow, D. Briscoe, M. Gillings, M. Holley, S. Smith, R. Leys, T. Silberbauer, C. Turnbull and A. Beattie, Antimicrobial defences increase with sociality in bees, Biological Letters 3 (2007), pp. 422-424.

Wille and Rutz, 1975 H. Wille and W. Rutz, Relationship between juvenile hormone titre and haemocytes in adult summer honeybees, Schweizerische Landwirtschaftliche Forschung 14 (1975), pp. 330-353.

Zuk and Stoehr, 2002 M. Zuk and A.M. Stoehr, Immune defense and host life history, The American Naturalist 160 (Suppl.) (2002), pp. S9-S22.

Corresponding author. Present address: Department of Entomology, University of Illinois at Urbana-Champaign, 320 Morill Hall, 505 S. Goodwin Avenue, Urbana, IL 61801, USA.

${ }^{1}$ These authors contributed equally to the work.

${ }^{2}$ Present address: Umeå Center for Molecular Pathogenesis, Umeå University, S-90187

Umeå, Sweden. 